\title{
Protective strategies for one-lung ventilation
}

\author{
Heezoo Kim \\ Department of Anesthesiology and Pain Medicine, Korea University College of Medicine, Seoul, Korea
}

Hypoxemia and acute lung injury (ALI) are major concerns with one-lung ventilation (OLV) during thoracic surgery. Hypoxemia is usually the result of alveolar hypoventilation and an increasing shunt fraction, whereas ALI is caused by ventilatory stress (volutrauma, atelectrauma, and barotrauma), reexpansion pulmonary injury, and the lung surgery itself. Various protective strategies can be used to overcome the challenges of OLV $[1,2]$. There is increasing interest in the impact of different ventilator modes on ALI and arterial oxygenation during OLV. Although there is some controversy, many studies report that pressure-controlled ventilation (PCV) has more favorable effects on respiratory function than volume-controlled ventilation (VCV) during thoracic surgery with OLV [3-5]. However, the tidal volume (TV) with PCV changes according to lung compliance. Consequently, PCV mode might not achieve the target $\mathrm{TV}$ in the case of patient with marked changes in compliance during anesthesia. Recently, new mechanical ventilation modes using a deceleration flow pattern with the Dräger (Auto-Flow ${ }^{\circledR}$ ) and Datex-Ohmeda (PCV-volume guaranteed [VG] mode) anesthetic machines were introduced. PCV-VG mode adjusts automatically and identifies the minimum inspiratory pressure needed to maintain the desired TV. In this ventilator mode, the TV was delivered consistently regardless of changes in the patient's compliance [6]. In this issue of the Korean Journal of Anesthesiology, Song et al. [7] compare the changes in airway pressure and arterial oxygenation between the VCV and PCV-VG ventilation modes during OLV. They demonstrated that PCVVG could attenuate airway pressure with a larger exhaled tidal volume rather than VCV during OLV in patients with relatively good pulmonary function. The authors recommend the use of
PCV-VG during OLV over VCV in terms of the peak inspiratory pressure and exhaled TV. The beneficial effects of PCV-VG on respiratory function during OLV are based on the enhanced arteriolar oxygenation and reduced lung damage due to pressure $[3,5]$. The advantages of arterial oxygenation with PCV-VG are explained by the decelerating flow with constant pressure, which reduces atelectasis, and the lowered inspiratory pressure reduces the lung damage and increases the homogenous distribution of the inspired gas [8].

However, PCV-VG mode could not always deliver a consistent TV. When the airway pressure reaches the maximum airway pressure limit preset to avoid barotrauma, the ventilator ends inspiration. Consequently, for a patient with markedly decreased compliance, PCV-VG mode does not provide sufficient TV because it also requires a high peak inspiratory pressure to deliver the desired TV. Therefore, it is important to maintain the compliance within an appropriate range by minimizing and preventing atelectasis during anesthesia, especially in OLV. The alveolar recruitment maneuver (ARM) with $5-10 \mathrm{cmH}_{2} \mathrm{O}$ positive endexpiratory pressure (PEEP) is a very useful, simple method for preventing atelectasis and promoting arteriolar oxygenation [9]. Various recruitment maneuvers have been developed, including high pressure-inflation for sustained seconds, intermittent sighs, and stepwise increases in the peak inspiratory pressure with PEEP $[9,10]$. However, the application of ARM itself is associated with a risk of lung injury and compromised hemodynamics. ARM, PEEP, and PCV-VG must be used meticulously to improve oxygenation based on the patient's lung condition and vital signs.

Corresponding author: Heezoo Kim, M.D., Ph.D., Department of Anesthesiology and Pain Medicine, Korea University College of Medicine, 148, Gurodong-ro, Guro-gu, Seoul 152-703, Korea. Tel: 82-2-2626-3236, Fax: 82-2-851-9897, E-mail: andigi@hanmail.net

(c) This is an open-access article distributed under the terms of the Creative Commons Attribution Non-Commercial License (http:// creativecommons.org/licenses/by-nc/3.0/), which permits unrestricted non-commercial use, distribution, and reproduction in any medium, provided the original work is properly cited. 


\section{References}

1. Lohser J. Evidence-based management of one-lung ventilation. Anesthesiol Clin 2008; 26: 241-72.

2. Karzai W, Schwarzkopf K. Hypoxemia during one-lung ventilation: prediction, prevention, and treatment. Anesthesiology 2009; 110: $1402-11$.

3. Tuğrul M, Camci E, Karadeniz H, Sentürk M, Pembeci K, Akpir K. Comparison of volume controlled with pressure controlled ventilation during one-lung anaesthesia. Br J Anaesth 1997; 79: 306-10.

4. Montes FR, Pardo DF, Charris H, Tellez LJ, Garzon JC, Osorio C. Comparison of two protective lung ventilatory regimes on oxygenation during one-lung ventilation: a randomized controlled trial. J Cardiothorac Surg 2010; 5: 99.

5. Yeom JH, Shin WJ, Kim YJ, Shim JH, Jeon WJ, Cho SY, et al. Comparison of volume-control and pressure-control ventilation during onelung ventilation. Korean J Anesthesiol 2009; 56: 492-6.

6. Keszler M. Volume-targeted ventilation. Early Hum Dev 2006; 82: 811-8.

7. Song SY, Jung JY, Cho MS, Kim JH, Ryu TH, Kim BI. Volume-controlled versus pressure-controlled ventilation-volume guaranteed mode during one-lung ventilation. Korean J Anesthesiol 2014; 67: 258-63.

8. Pu J, Liu Z, Yang L, Wang Y, Jiang J. Applications of pressure control ventilation volume guaranteed during one-lung ventilation in thoracic surgery. Int J Clin Exp Med 2014; 7: 1094-8.

9. Tusman G, Bohm SH, Vazquez de Anda GF, do Campo JL, Lachmann B. 'Alveolar recruitment strategy' improves arterial oxygenation during general anaesthesia. Br J Anaesth 1999; 82: 8-13.

10. Shin CS, Chang $\mathrm{CH}$, Koh SO. The effects of repetitive alveolar recruitment on oxygenation and compliance in ARDS patients. Korean J Anesthesiol 2007; 52: S66-S71. 